\title{
Performance evaluation of ad hoc routing protocols in VANETs
}

\author{
Mohammed ERRITALI, Bouabid El Ouahidi \\ laboratory of computer science research \\ Department of Computer Science \\ Mohamed V University - Faculty of Sciences Rabat, Morocco
}

\begin{abstract}
The objective of this work is to compare with simulating, using OPNET the performance of five Ad hoc routing protocols: DSR, AODV, OLSR, TORA and GRP, and to examine the impact of mobility and the density of nodes on the behavior of these protocols in a Vehicular Ad hoc NETwork (VANET). The results show that there is not a protocol that is favorite for all evaluation criteria. Indeed, each protocol has different behavior in relation to performance metrics considered, including the rate of routing packets sent, delay, and the debit.
\end{abstract}

Keywords-Routing protocols; OPNET; Performance evaluation; DSR; AODV; OLSR; TORA; GRP.

\section{INTRODUCTION}

The VANETs (Vehicular Ad hoc NETworks) [1,2] are a new form of mobile ad hoc networks used to establish communications between vehicles or with infrastructure located on roadsides. They are characterized by a dynamic topology and are generally ad hoc networks, that is to say without management and control infrastructure of communication.

These networks are used to meet the needs of communication applied to transportation networks [1,2] to improve the driving and road safety for road users.

In a VANET network routing is an important mechanism for finding and maintaining a communication path between a pair of remote nodes.

In recent years, the improved performance of routing mechanisms in ad hoc networks was one of the main issues. This is reflected by the appearance of hundreds of protocols in the literature $[3,4,5,6,7,8,9,10,11,12,13]$ of which only a few have been subjected to standardization.

For the evaluation of these protocols simulation offers a vision on the performance of the protocol itself before adopting and commercializing by a organization of standardization and brings us closer to the real use of the protocol, that helps us to select the best protocols that have a good behavior in different scenario, the simulation also allows the developer to test their protocols routing algorithms to improve them.

The performance metrics of ad hoc protocols have been discussed in the first RFC published by the MANET working group [15], by separating these metric to external metrics measurement (how the performance of the protocol is perceived by mechanisms using routing) and internal measurement (for the same level of external performance, two algorithms will deploy different volumes overhead).

External measures the effectiveness of an ad hoc protocol includes:

- The delay

- The debit

- The acquisition time of a road that is only valid for reactive protocols.

- The percentage of segments received out of sequence

To measure the internal efficiency of the protocol, the following metrics are recommended:

- The average number of bits of data transmitted / number of data bits received

- The average number of control bits transmitted / number of data bits received

- The average number of control packets and data transmitted / number of data packets received.

The purpose of this paper is to study the performance of ad hoc routing protocols to meet the following three questions:

1) What are the main differences between the ad hoc routing protocols?

2) What routing protocol provides better performance in vehicular ad-hoc networks?

3) What are the factors that influence the performance of these routing protocols?

In attempting to answer the above questions, we used OPNET [14] to compare the performance of the five protocols AODV, DSR, TORA, OLSR and GRP to examine the impact of mobility and density of nodes on the behavior these protocols regarding the speed, the traffic of routing packet sent, the debit and delay.

\section{AD HOC ROUTING PROTOCOLS}

Routing is the mechanism that allows the nodes of a vehicular network to communicate continually with each other information about the topology and link state in order to determine the best routes. 
Figure 1 illustrates the taxonomy of these routing protocols which can be classified as topology-based and geographic (position-based) in VANET.

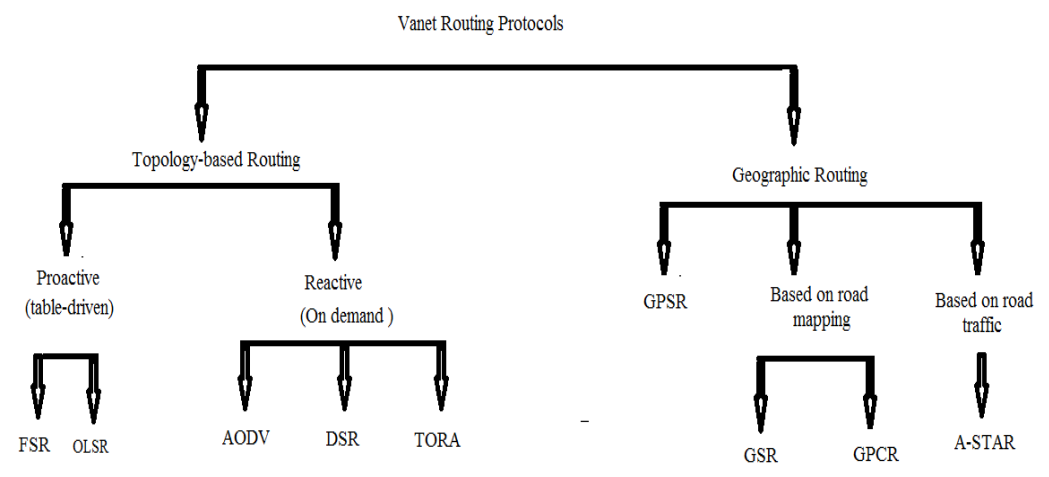

Fig.1. VANET routing Protocols

\section{A. Topology-based Protocols:}

These routing protocols use the link information which exists in the network to send the data packets from source to destination. They can also be classified as active (table-driven) and reactive (on demand) routing.

\section{1) Proactive (table-driven):}

Proactive protocols are used to establish the routes in advance on the basis of the periodic exchange of routing tables.

\section{a) Fisheye State Routing (FSR)}

FSR [3] is a proactive protocol link state. It assumes that a faraway topology change has not a significant influence on the calculation of the route locally. Therefore, the exchange of routing table updates is a function of the distance. More a node is faraway less it receives local topology updates.

FSR does not flood the network with updates, but all nodes periodically exchange with its neighbors partial routing update information. Indeed, all links propagates hop by hop in each sends.

FSR has a complete map of the network but cannot guarantee accuracy of all connections between farther nodes.

\section{$(O L S R)$}

b) The protocol Optimized Link State Routing Protocol

OLSR [4] is a link-state protocol, which optimizes the way of broadcast of control messages to save bandwidth consumption through the use of the concept of "multipoint relays" (MPRs) shown Figure 2 in which each node selects a subset of its neighbors to forward its packets when broadcast.

Based on the distribution using the MPRs, all nodes in the network are achieved with fewer repetitions. A MPR set of node $\mathrm{N}$ is the minimal set of its 1-hop neighbors that cover (in the direction of the communication range) two-hop neighbors.

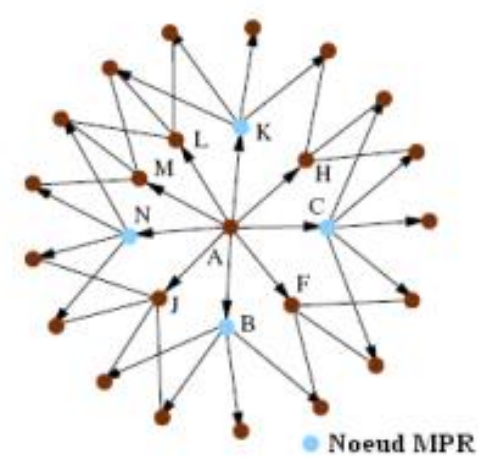

Fig.2. Multipoint relays in OLSR

\section{2) Reactive (On Demand)}

Reactive protocols, only keep the roads in use for routing. On demand at the time of packet routing, the protocol will search through the network a route to the destination. The conventional method of route lookup is to flood the network with a query, in order to find the target station, which responds with the reverse path.

\section{a) Dynamic Source Routing (DSR)}

The DSR protocol [5] is based on the "source routing", which means that the path traveled by the packet is included in the header of the data packet from the source to be read by routers.

The DSR protocol is composed of two distinct mechanisms: the first is used to find routes on demand, and the second is responsible for the maintenance of the communication route in progress.

The limitation of this protocol is that the route maintenance process does not locally repair a broken link and the performance of the protocol decreases with increasing mobility.

\section{b) Ad Hoc On Demand Distance Vector (AODV)}

AODV [6] is a reactive protocol based on the principles of distance vector routing protocols. It borrows its mechanisms in DSR discovery and maintenance of routes and uses a hop by hop routing, and sequence numbers.

AODV builds routes by using a series of queries "route request / route reply".

When a source node wants to establish a route to a destination for which it does not already have a route, it broadcasts a packet "route request" (RREQ) throughout the network. Nodes receiving this packet update their information for the source and set pointers of return to the source in the routing tables. In addition to the IP of the source, the current sequence number and a broadcast identifier, the RREQ also contains the sequence number of the destination most recent known by the source. A node receiving a RREQ packet will then emit a "route reply" (RREP) if it is the destination or if it has a route to the destination with a sequence number greater than or equal to that included in the RREQ. If this is the case, it sends a RREP packet to the source. Otherwise, it re-broadcasts the RREQ. Nodes keep track of each source IP and identifiers 
of RREQ broadcast packets. If they receive a RREQ which they have already dealt with, they remove it.

\section{c) Temporally Ordered Routing Protocol (TORA)}

TORA [7] algorithm belongs to the class of algorithms (Link Reversal). It is based on the principle algorithms that try to maintain the property called "destination orientation" directed acyclic graphs (DAG). A directed acyclic graph is oriented destination if there is still a possible path to a specified destination. The graph is undirected destination if at least one link becomes faulty. In this case, the algorithms use the concept of reverse links. This concept ensures the transformation of the previous paragraph in a directed graph destination in a finite time. To maintain the DAG oriented destination, the TORA algorithm uses the concept of node size. each node has a size that exchange it with all of its immediate neighbors. This new concept is used in the orientation of the network links. A link is always directed from the node to the larger to the smaller node size.

The protocol is based on three phases: the creation of routes, maintenance of routes and clearing of route using three separate packets QRY, UPD and CLR.

TORA adapts to the mobility of vehicular networks by storing multiple paths to the same destination, so that many topology changes will not affect the routing of data, unless all paths that lead to destination are lost.

\section{B. Position based protocols:}

It is a routing taking into account the geographical position [8] of the nodes. To perform a geographic routing in an ad hoc network, it is essential that: all nodes have location means: via native system such as GPS, and that the source node knows the position of the destination node.

Various solutions have been proposed. Among these:

a) Greedy Perimeter Stateless Routing (GPSR) :

The GPSR [2,9,10] algorithm uses the position of the nodes in addition to the destination to determine the next node to which retransmit the packet. By default, a node GPSR uses greedy mode and forwards the packet to the neighbor closest to the destination. The Neighborhood detection is done by periodic diffusion of Beacon messages containing the address of the node and its position (x, y).

\section{b) Geographic Source Routing (GSR)}

GSR [2,11] is based on the geographical position and the information about the road topology in order to build a knowledge adapted to the urban environment. According to the protocol GSR, a source vehicle wishing to send a data packet to a target vehicle, calculates the shortest routing path to reach this target vehicle using geographical information of road map. We note that the routing path in question is calculated in its entirety, using for example the algorithm Djikstra. From the calculated routing path, the vehicle source selects a sequence of intersections through which the data packet must travel to reach the target vehicle. This sequence of intersections is formed by a set of fixe geographical points of data packet passage. And to send messages from one intersection to another, the authors propose to use a greedy approach. c) Greedy, Perimeter Coordinator Routing (GPCR) :

GPCR [2,12] is a combination of protocol GPSR and the use of road mapping. The authors assume that each node can know whether if it is in intersection which case it acquires the status of a coordinator node. Thus, the messages are transmitted along the road using a greedy approach with preference given to coordinators nodes.This means that choosing of next relay node, a node coordinator (a node at an intersection), is preferred to a non-coordinator node, even if it is not the closest to the destination, and this to avoid obstacles radios.

\section{$S T A R)$}

d) Anchor-based Street and Traffic Aware Routing (A-

A-STAR [2,13] is a routing protocol based on the geographical position of Metropolitan vehicular environment. It uses special information on city bus routes to identify an anchor path with high connectivity for packet forwarding. ASTAR protocol is similar to the GSR by adopting an approach based routing anchor that reflects the characteristics of the streets. However, unlike GSR it calculates the "anchor paths" depending on the traffic. A weight is assigned to each street according to its capacity (large or Small Street).

\section{THE METRICS INCLUDED IN OUR SIMULATION}

\section{A. The routing traffic}

Routing traffic sent by each node is an important measure of scalability of routing protocol, and therefore the network. It is defined as the total number of routing packets transmitted over the network, and is expressed in bits per second or packets per second, this number is recalculated in each transmitting or receiving packets. This test is done to see if the network is loaded or not. Routing traffic is expressed by the following equation:

Traffic routing $=$ number of packets-(number of received packets +number of packets lost)

This metric is used to select the protocol that encumbers the least network.

\section{B. The debit}

The debit is the transfer rate at a given time, it is expressed in bits per second. The factors that affect the debit in VANET are frequent topology changes and limited bandwidth.

\section{C. delay}

The delay is the time required for a packet to arrive at the destination, ie: the time that elapses between the generation of packet source and its receipt by the application layer of the destination. For each packet in the network this period is calculated as follows:

delay $=$ time of receipt of package - transmission time of the packet (2)

\section{MODELING OF SIMULATION AND SCENARIOS}

Our goal in this section is to model the behavior of routing protocols under different conditions of load and speed mobility in vehicles to infrastructure communications (V2I) [2] to 
examine the statistical averages of debit, delay, and the routing traffic generated in the entire VANET network .

We modeled the network with a determined surface area. Nodes (vehicles) and the server were randomly distributed in the geographic area. In the simulations, we used the TCP traffic to study the effects of ad hoc protocols on TCP-based applications such as web and file transfer.

We have configured an FTP application profile in our study where the nodes are a WLAN mobile clients with a debit fixed at $11 \mathrm{Mbps}$, while the destination is a server of a wireless network also with a debit of $11 \mathrm{Mbps}$.

We also used the mobility model " random waypoint " widely accepted to describe mobility behavior of VANET nodes.

We also selected two-speed mobility, the first is $10 \mathrm{~m} / \mathrm{s}$ $(36 \mathrm{Km} / \mathrm{h})$, which reflects a low mobility, and the second is $28 \mathrm{~ms} / \mathrm{s}(100.8 \mathrm{~km} / \mathrm{h})$, which reflects the high mobility of nodes.

For the density of the network we have chosen the number of nodes: 5 for a reliable density, 20 for a medium density and 40 for a high density.

Table 1 below shows the 30 scenarios used in our simulation. For each routing protocol we proceed to 6 scenarios, each scenario is characterized either by a change in the mobility speed or the number of nodes:

TABLE I. Scenarios Used

\begin{tabular}{|l|l|l|l|}
\hline Scenarios & $\begin{array}{l}\text { Nombre } \\
\text { des } \\
\text { nœuds }\end{array}$ & $\begin{array}{l}\text { Vitesse de } \\
\text { mobilité } \\
(\mathrm{m} / \mathrm{S})\end{array}$ & $\begin{array}{l}\text { Protocole } \\
\text { utilisé }\end{array}$ \\
\hline Scenario 1 & 5 & 10 & DSR \\
\hline Scenario 2 & 5 & 28 & DSR \\
\hline Scenario 3 & 20 & 10 & DSR \\
\hline Scenario 4 & 20 & 28 & DSR \\
\hline Scenario 5 & 40 & 10 & DSR \\
\hline Scenario 6 & 40 & 28 & DSR \\
\hline Scenario 7 & 5 & 10 & AODV \\
\hline Scenario 8 & 5 & 28 & AODV \\
\hline Scenario 9 & 20 & 10 & AODV \\
\hline Scenario 10 & 20 & 28 & AODV \\
\hline Scenario 11 & 40 & 10 & AODV \\
\hline Scenario 12 & 40 & 28 & AODV \\
\hline Scenario 13 & 5 & 10 & TORA \\
\hline Scenario 14 & 5 & 28 & TORA \\
\hline Scenario 15 & 20 & 10 & TORA \\
\hline Scenario 16 & 20 & 28 & TORA \\
\hline Scenario 17 & 40 & 10 & TORA \\
\hline Scenario 18 & 40 & 28 & TORA \\
\hline Scenario 19 & 5 & 10 & OLSR \\
\hline Scenario 20 & 5 & 28 & OLSR \\
\hline Scenario 21 & 20 & 10 & OLSR \\
\hline Scenario 22 & 20 & 28 & OLSR \\
\hline Scenario 23 & 40 & 10 & OLSR \\
\hline Scenario 24 & 40 & 28 & OLSR \\
\hline & & & \\
\hline & & & \\
\hline
\end{tabular}

\begin{tabular}{|l|l|l|l|}
\hline Scenario 25 & 5 & 10 & GRP \\
\hline Scenario 26 & 5 & 28 & GRP \\
\hline Scenario 27 & 20 & 10 & GRP \\
\hline Scenario 28 & 20 & 28 & GRP \\
\hline Scenario 29 & 40 & 10 & GRP \\
\hline Scenario 30 & 40 & 28 & GRP \\
& & & \\
\hline
\end{tabular}

\section{RESULTS AND INTERPRETATION}

In this section, we will discuss and analyze the results of our simulations of routing protocols used, starting with an analysis of routing traffic sent in the network, then the delay and finally the network debit.

\section{a) Routing traffic}

According to Figures 3, 4, 5, 6, 7, 8 we note for the DSR protocol, in all scenarios that mobility does not affect the traffic in the case of 5 nodes it is estimated by 10 bits / sec in the case of nodes 20 it is $200 \mathrm{bits} / \mathrm{sec}$ and in the case of nodes 40 it is 500 bits / s.

Regarding the protocol AODV we notice the same thing, mobility does not have any influence on the traffic.

The TORA protocol knows a decrease in routing traffic in the case of 5 and 20 nodes with different mobility speed and stabilizes with a traffic of 425 bits / sec to 5 nodes and 6,000 bits / sec to 20 nodes.

With more nodes and high speed mobility: TORA generates a big traffic.

For OLSR mobility does not affect routing traffic but increases with the network load and is almost at the same level in all scenarios, with a $1150 \mathrm{bit} / \mathrm{sec}$ in the case of a network of 5 nodes, 9000 bits / sec for the network of 20 nodes and 30000 bits / sec for high network load estimated withe 40 nodes. For the routing protocol GRP we note that traffic routing rapidly decreases and remains almost stable and weak the entire duration of the simulation for all scenarios, traffic is estimated by 550 bits / sec in the case of 5 nodes and 2000 bits / sec in the case of 20 and 40 nodes.

Our simulation shows that the geographic routing GRP does not generate a large traffic routing in comparison with TORA and OLSR protocols that use control messages to detect topology changes in their neighborhood.

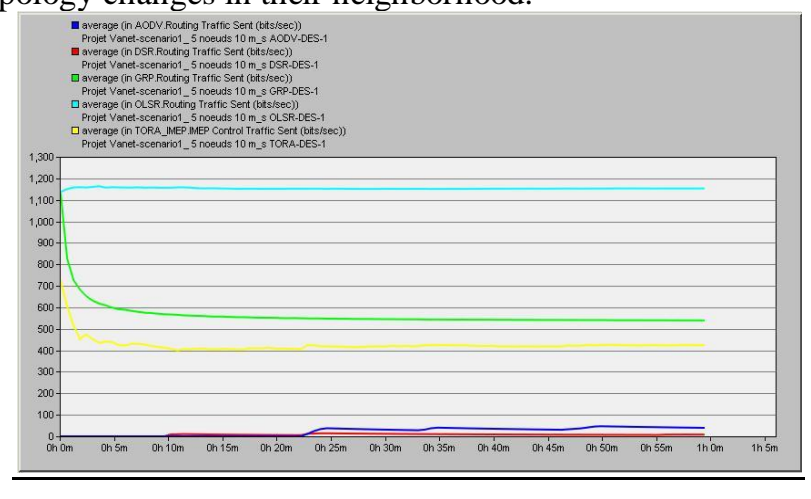

Fig.3. Routing traffic of 5 nodes and mobility speed of $10 \mathrm{~m} / \mathrm{s}$. 


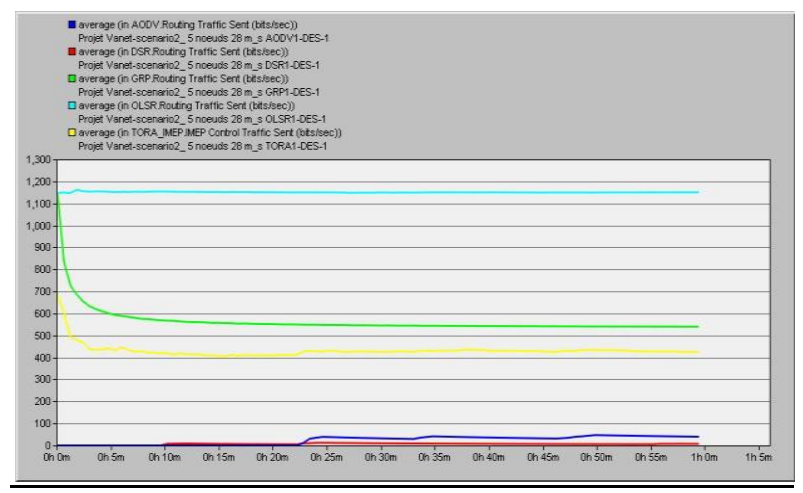

Fig.4. Routing traffic of 5 nodes and mobility speed of $28 \mathrm{~m} / \mathrm{s}$.

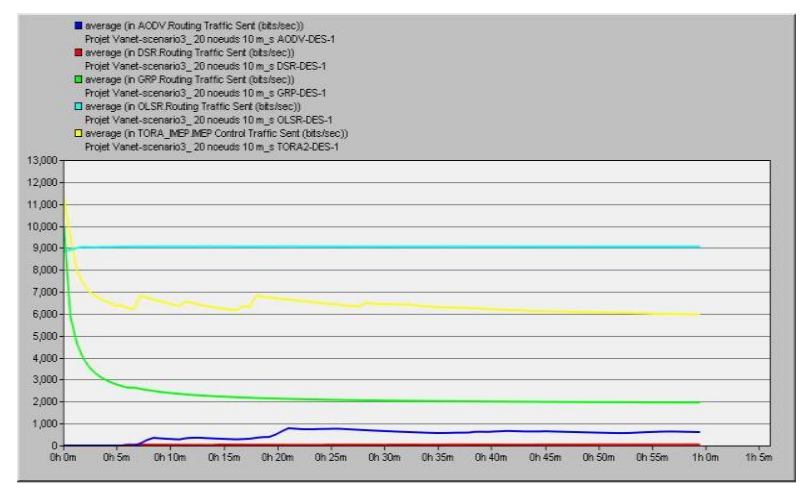

Fig.5. Routing traffic of 20 nodes and mobility speed of $10 \mathrm{~m} / \mathrm{s}$.

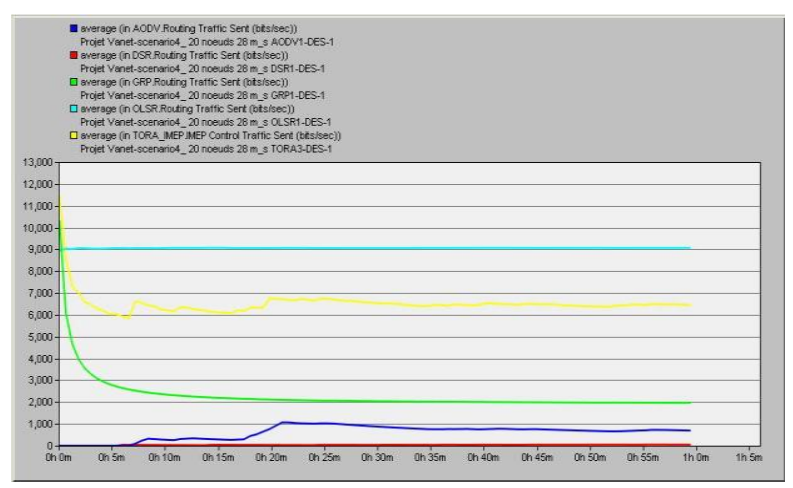

Fig.6. Routing traffic of 20 nodes and mobility speed of $28 \mathrm{~m} / \mathrm{s}$.

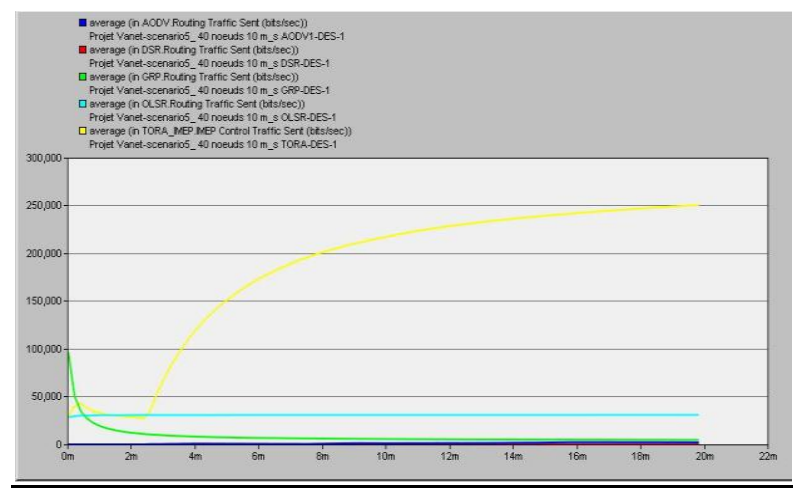

Fig.7. Routing traffic of 40 nodes and mobility speed of $10 \mathrm{~m} / \mathrm{s}$.

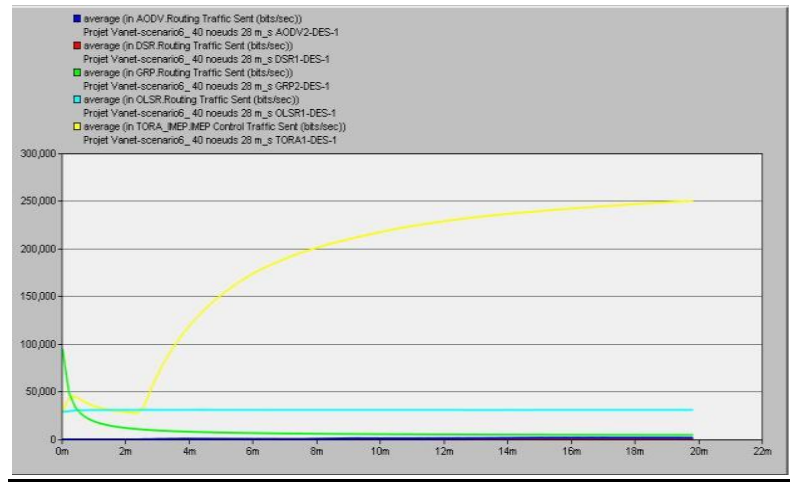

Fig.8. Routing traffic of 40 nodes and mobility speed of $28 \mathrm{~m} / \mathrm{s}$.

\section{b) The delay}

According to figures 9,10,11,12,13, 14 in all scenarios, we observe that OLSR has the lowest delay that varies between $0.0003 \mathrm{sec}$ and $0.0004 \mathrm{sec}$. OLSR is a proactive routing protocol, which means that the network connections are always ready every time when the application layer has a traffic to send. The periodic updates keep the routing paths available for use and the absence of a high latency induced by the discovery process routes in OLSR explains its relatively low delay. With a larger number of mobile nodes, the performances of this protocol are in competition with those of AODV protocol with a delay of $0.0005 \mathrm{sec}$.

For small networks with 5 nodes, we observe that TORA has a delay of $0.0013 \mathrm{sec}$ and outperforms DSR, which has a delay of $0.0033 \mathrm{sec}$ in the two case of mobility. On the other hand, TORA is in concurrence with AODV in the case of low speed where they have almost the same delay estimated by $0.0013 \mathrm{sec}$ but the TORA protocol outperforms AODV in the case of high speed with a delay of $0,0070 \mathrm{sec}$.

When the number of nodes is growing, we note that TORA suffers from a significant deterioration in his delay. One reason for this deterioration is the process of route discovery. The AODV protocol also has a very low delay estimated by 0,0013 $\mathrm{sec}$ in the case of 5 nodes and $0.0005 \mathrm{sec}$ in the case of 20 nodes, and it happens the second after OLSR. This is observed in all scenarios, except in the case of reducing the number of nodes and high speed where it outperforms TORA. However, 
we observe that the performance of AODV protocol improves with increasing the number of nodes.

DSR shows a uniform delay in the case of the two speed mobility with 5 and 20 nodes where it is $0.0033 \mathrm{sec}$ and increases in the case of 40 nodes has $0 / 0070 \mathrm{sec}$.

The DSR protocol uses cached routes and often it sends traffic on obsolete routes, which can cause retransmissions and cause excessive delays.

The GRP protocol outlines a more coherent delay than other protocols ranging between 0.0004 and 0.0005 in all scenarios.

In conclusion, we observed that OLSR exhibits a very low delay in all scenarios. While for the TORA protocol we note that it has a big delay in a network with a big traffic, and that the mobility did not affect his delay.

Regarding the AODV protocol, we notice an improvement in delay when the network size increases and that the speed does not affect the delay, and finally the DSR protocol adopts a coherent approach and undergoes further delay when the network grows but the speed of mobility does not have profound effects on its performance.

The three reactive protocols had high delays due to the increasing demands of route discovery, unlike the geographic routing, which exposes an important delay only in the case of a less loaded network.

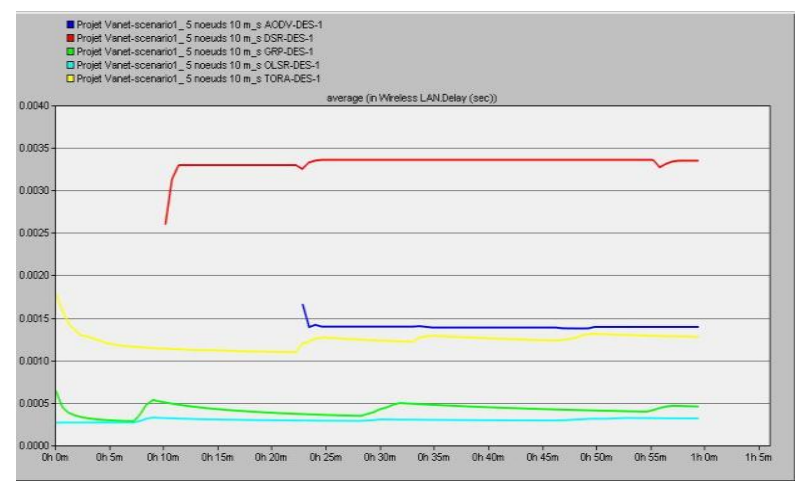

Fig.9. Delay of 5 nodes and mobility speed of $10 \mathrm{~m} / \mathrm{s}$.

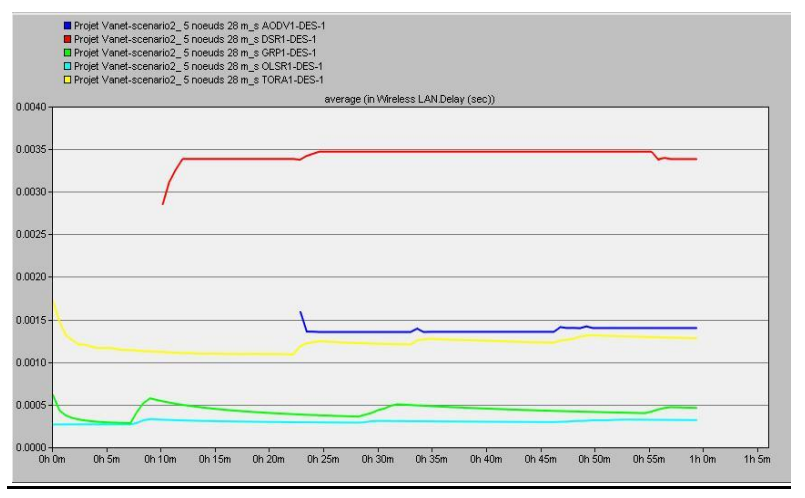

Fig.10. Delay of 5 nodes and mobility speed of $28 \mathrm{~m} / \mathrm{s}$.

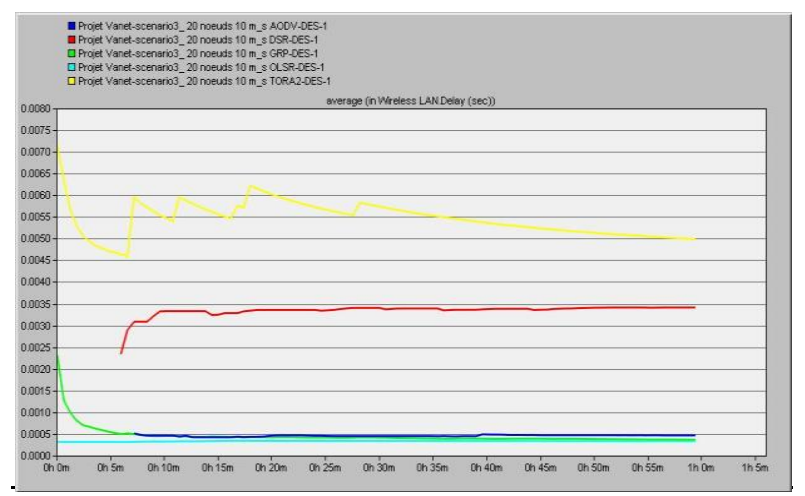

Fig.11. Delay of 20 nodes and mobility speed of $10 \mathrm{~m} / \mathrm{s}$.

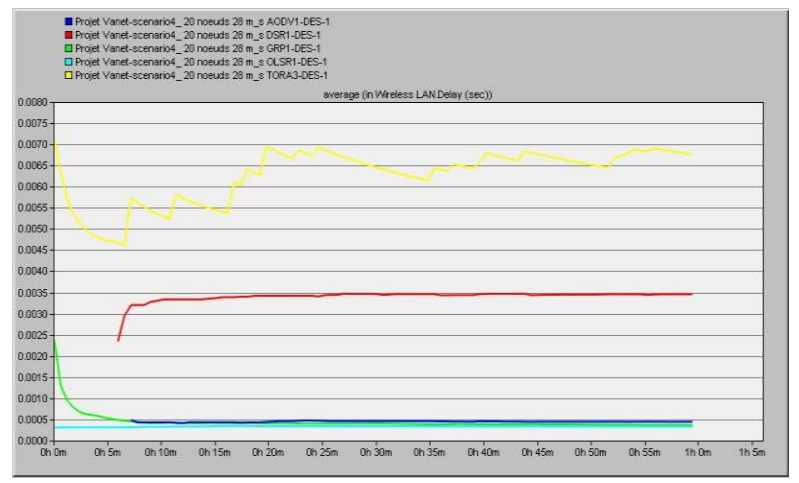

Fig.12. Delay of 20 nodes and mobility speed of $28 \mathrm{~m} / \mathrm{s}$.

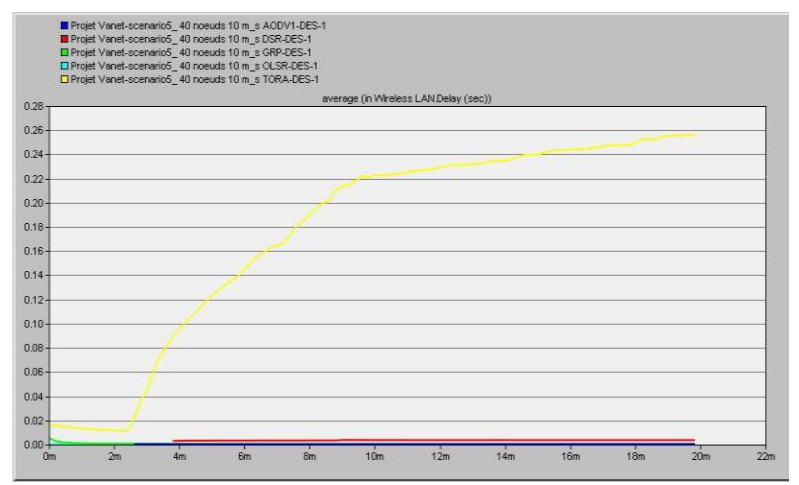

Fig.13. Delay of 40 nodes and mobility speed of $10 \mathrm{~m} / \mathrm{s}$. 


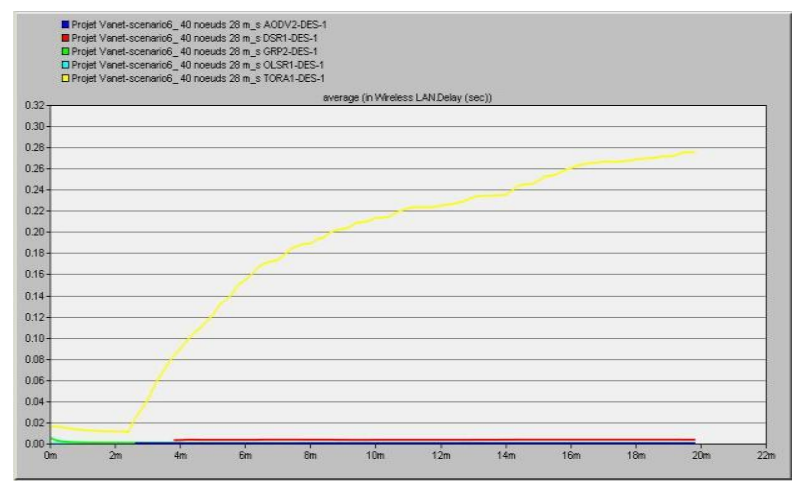

Fig.14. Delay of 40 nodes and mobility speed of $28 \mathrm{~m} / \mathrm{s}$.

c) The debit

According to Figures 15, 16, 17, 18, 19, 20 we observe that the OLSR protocol has a very significant debit where it exceeds $12,000 \mathrm{bits} / \mathrm{sec}$.

In the network with 5 nodes, DSR has a debit of 4700 bits / sec and outperforms TORA which has a debit of 3500 bits / sec and AODV which has a debit of 3000 bits / sec at the two case of mobility speed.

The AODV protocol has a lowest debit about 20,000 bits / s When the network are composed with a small number of nodes that move with low speed, and It has a big debit approximately $100,000 \mathrm{bits} / \mathrm{sec}$ in the case of a higher speed mobility in larger networks.

OLSR has a constant debit in the case of two mobility speed where it exceeds $12,000 \mathrm{bits} / \mathrm{sec}$.

The debit of TORA protocol Increases in the case of high mobility to reach 400,000 bits / sec.

For the GRP protocol we notice that de debit decreases on the Beginning of the simulation and then remains almost stable but for a network of 5 nodes with the two mobility speed the debit varies According to a form closer to a sine function around $6800 \mathrm{bits} / \mathrm{sec}$, and remains significant in all phases of the simulation.

According to our simulation we notice that the geographic routing has better debit compared to DSR, AODV and TORA.

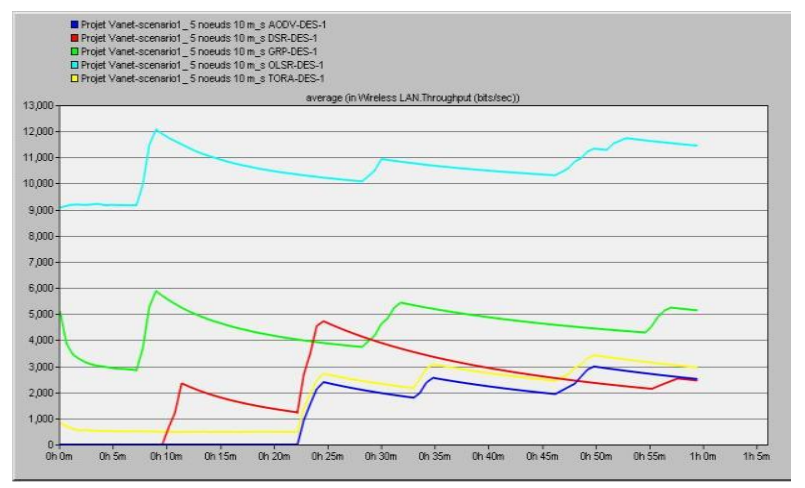

Fig.15. Debit of 5 nodes and mobility speed of $10 \mathrm{~m} / \mathrm{s}$

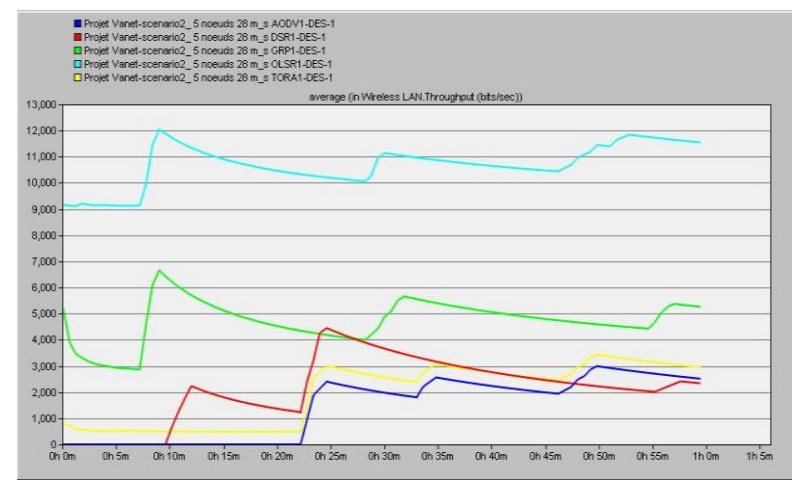

Fig.16. Debit of 5 nodes and mobility speed of $28 \mathrm{~m} / \mathrm{s}$

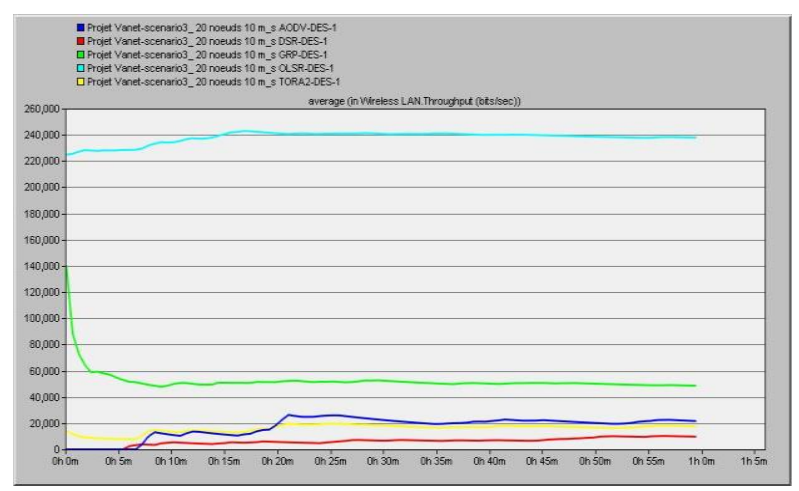

Fig.17. Debit of 20 nodes and mobility speed of $10 \mathrm{~m} / \mathrm{s}$

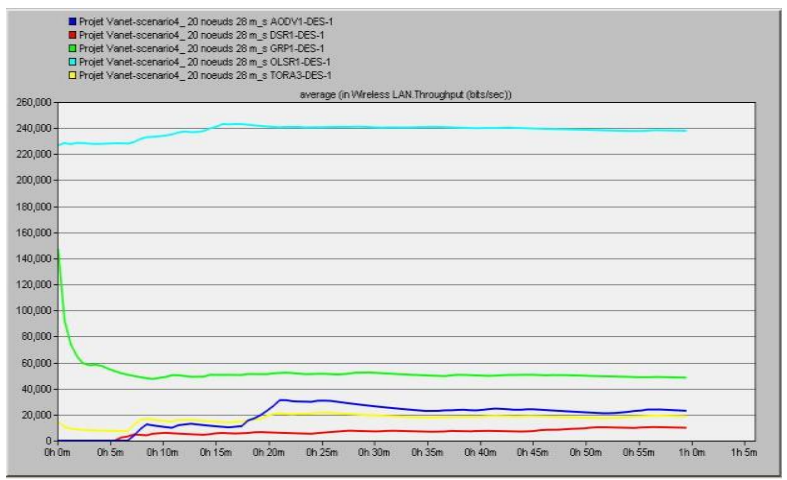

Fig.18. Debit of 20 nodes and mobility speed of $28 \mathrm{~m} / \mathrm{s}$

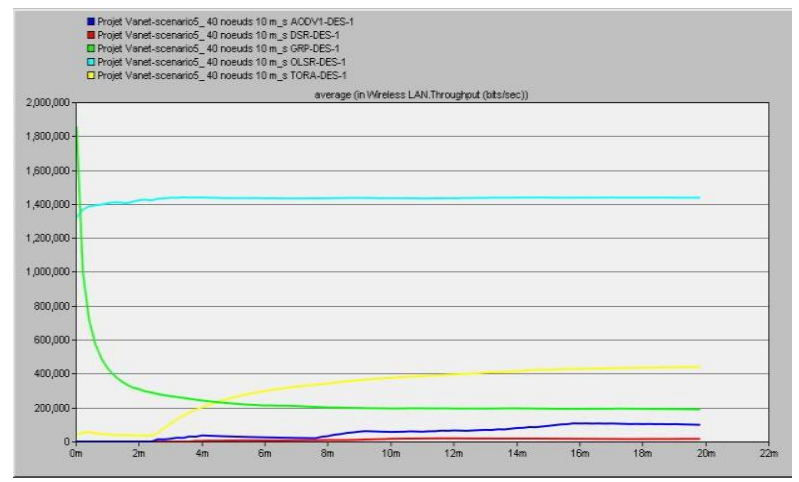

Fig.19. Debit of 40 nodes and mobility speed of $10 \mathrm{~m} / \mathrm{s}$ 


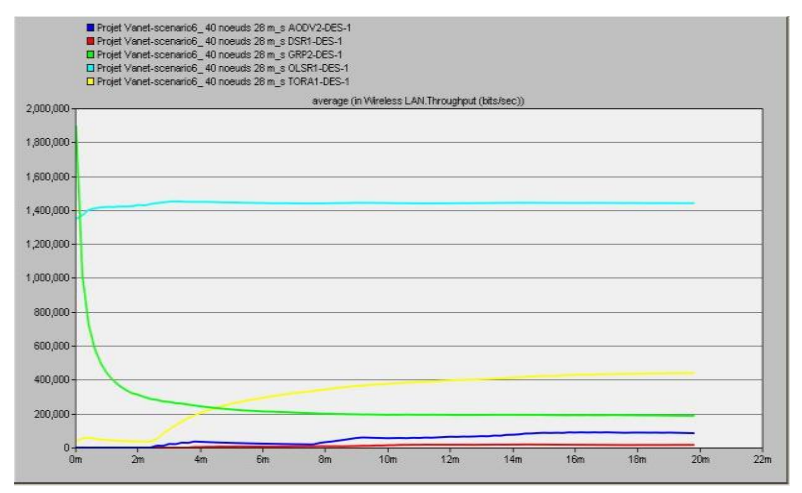

Fig.20. Debit of 40 nodes and mobility speed of $28 \mathrm{~m} / \mathrm{s}$

\section{CONCLUSION}

In this paper we have presented a comparative study of five protocols AODV, DSR, TORA, OLSR and GRP regarding the debit, the routing traffic sent and delay, the table below summarizes the characteristics noted in our simulation.

TABLE II. Comparison Of Routing Protocols

\begin{tabular}{|c|l|}
\hline The protocol used & \multicolumn{1}{|c|}{ Conclusions } \\
\hline \multirow{5}{*}{ OLSR } & $\begin{array}{l}\text { OLSR outperforms AODV, } \\
\text { DSR and TORA in debit and } \\
\text { delay. } \\
\text { OLSR has the worst } \\
\text { performance in routing traffic } \\
\text { generated. It is therefore well } \\
\text { suited for high debit networks. } \\
\text { High traffic routing shows } \\
\text { that OLSR is not suitable for } \\
\text { low debit networks. }\end{array}$ \\
\hline DSR & $\begin{array}{l}\text { DSR is the best candidate in } \\
\text { networks of low debit and } \\
\text { medium in size. } \\
\text { However, it has a large traffic } \\
\text { routing in the case of long } \\
\text { paths (large networks). It } \\
\text { inserts in the packet IP } \\
\text { addresses of all nodes in the } \\
\text { loute it uses. }\end{array}$ \\
\hline $\begin{array}{l}\text { AODV is suitable for } \\
\text { networks of low and medium } \\
\text { load with low speed mobility. }\end{array}$ \\
\hline AODV & \begin{tabular}{l} 
TORA is suitable for small \\
networks. It loses its \\
performance under rapid \\
expansion. \\
\hline TORA
\end{tabular} \\
\hline
\end{tabular}

GRP

GRP significantly reduces the signaling (control packets), especially in large and dynamic networks.

Simplicity and low memory requirements. More suited to large networks.

We found in this study that geographic routing is best suited for highly dynamic vehicular networks because of its low latency and low amount of routing traffic.

The problem remains in the ability to choose metrics that provide appropriate results to both vehicular environments (cities, highways), which have different characteristics.

\section{REFERENCES}

[1] S. Khalfallah, M. Jerbi, M. O. Cherif , S.M. Senouci , Bertrand Ducourthial, Expérimentations des communications inter-véhicules, Colloque Francophone sur l'Ingénierie des Protocoles (CFIP), Les Arcs : France (2008).

[2] M.JERBI, Protocoles pour les communications dans les réseaux de véhicules en environnement urbain : Routage et GeoCast basés sur les intersections . Thèse, France (2008).

[3] Bijan Paul, Md. Ibrahim, Md. Abu Naser Bikas, VANET Routing Protocols: Pros and Cons, International Journal of Computer Applications (0975 - 8887), Volume 20- No.3, April 2011.

[4] Thomas Clausen and Philippe Jacquet. IETF RFC3626 : Optimized link state routing protocol (OLSR). October 2003.

[5] D.B. Johnson, D.A. Maltz, Y-C. Hu, The Dynamic Source Routing Protocol for Mobile Ad Hoc Networks (DSR), Internet Draft : <draftietf-manetdsr-10.txt>, 19 Juillet 2004.

[6] C.E. Perkins, E.M. Belding-Royer, and S. Das, Ad Hoc On Demand Distance Vector (AODV) Routing, IETF RFC 3561, 2003.

[7] V. Park, S. Corson, "Temporally-Ordered Routing Algorithm (TORA) Version 1 Functional Specification", IETF Internet draft, work in progress, draft-ietf-manet-tora-spec-04.txt, 2001.

[8] Giordano S., et al., "Position based routing algorithms for ad hoc networks: A taxonomy," in Ad Hoc Wireless Networking, X. Cheng, X. Huang and D.Z. Du (eds.), Kluwer, December 2003.

[9] Karp, B. and Kung, H.T., Greedy Perimeter Stateless Routing for Wireless Networks, inproceedings of the Sixth Annual ACM/IEEE International Conference on Mobile Computing and Networking (MobiCom 2000), Boston, MA, August, 2000.

[10] Young Jin Kim and Ramesh Govidan ,Greedy Perimeter Stateless Routing (GPSR) Detailed-level Design Specification (DDS) Version 0.1 , Department of Computer Science University of Southern California, (July 6, 2003).

[11] C. LOCHERT, H. HARTENSTEIN, J. TIAN, D. HERRMANN, H. Füßler, M. MAUVE, "A Routing Strategy for Vehicular Ad Hoc Networks in City Environments", IEEE Intelligent Vehicles Symposium (IV2003), pp. 156-161, Columbus, OH, USA,( June 2003).

[12] C. LOCHERT, M. MAUVE, H. Füßler, H. HARTENSTEIN, "Geographic Routing in City Scenarios", MobiCom (2004).

[13] B.-C. SEET, G. LIU, B.-S. LEE, C. H. FOH, K. J. WONG, K.-K. Lee, "A-STAR: A Mobile Ad Hoc Routing Strategy for Metropolis Vehicular Communications". NETWORKING 2004, 989-999.

[14] Opnet, http://www.opnet.com/

[15] Corson (S.) et Macker (J.). - Mobile Ad hoc Networking (MANET) : Routing Protocol Performance Issues and Evaluation Considerations. RFC n2501, IETF, ( janvier 1999). 